\title{
DESCONFORTO E DOR DURANTE REALIZAÇÃO DA MAMOGRAFIA
}

\author{
Ruffo freitas Júnior*, Walsir faganelo fiori, francisco José de faria Ramos, Eduardo Godinho, Rosemar Macedo Sousa Rahal, \\ Jayme Gonçalves de Oliveira
}

Trabalho realizado nos serviços de Ginecologia e Mama e de Radiologia da Associação de Combate ao Câncer em Goiás; nos departamentos de Ginecologia e Obstetrícia e de Anatomia Patológica e Imagenologia da Faculdade de Medicina da Universidade Federal de Goiás; na Clínica Radiológica de Goiânia; na Clínica São Camilo e na Clínica Aro, Goiânia, GO

\section{*Correspondência:}

Alameda das Rosas, 533, S.

Oeste,

74110-060, Goiânia, GO

ruffoir@terra.com.br

\section{RESUMO}

A mamografia é o método mais importante na detecção precoce do câncer de mama. Não obstante, o desconforto e a dor são queixas freqüentes durante o exame.

ОвјEтIvo. Avaliar a freqüência de desconforto e de dor durante a mamografia e identificar fatores relacionados com essas queixas.

Métodos. Estudo prospectivo incluindo 2.164 pacientes, sendo que 996 previdenciárias e 1.168 da rede privada. Após o exame, a paciente quantificava a dor sentida, de acordo com uma escala linear analógica. 0 desconforto foi avaliado qualitativamente por análise multivariada.

Resultados. O desconforto foi a queixa de $90 \%$ das pacientes; dessas 12\% referiram desconforto intenso ou intolerável. Apenas $2 \%$ do grupo estudado não apresentaram dor durante o exame. Os fatores associados à dor foram: idade da paciente, o uso de anticoncepcional (ACO), mastalgia prévia e pacientes privadas. O desconforto esteve independentemente associado ao centro de realização do exame $(O R=2,50 ;|C|, 64-5, \mid 7)$ e à mastalgia prévia $(O R=3,|5 ;| C|, 96-7| 2$,$) . A aceitabilidade$ de uma futura mamografia foi de $98 \%$.

Conclusäo. Mulheres jovens ( $<50$ anos), usuárias de ACO, portadoras de mastalgia e provenientes de serviços privados apresentaram maior intensidade de dor e desconforto durante a realização da mamografia.

UnITERMOS: Mama. Mamografia. Câncer. Rastreamento. Dor. Desconforto.

\section{INTRODUÇÃO}

A mamografia tem sido cada vez mais empregada no diagnóstico das lesões de mama e, dentre os métodos de detecção precoce para o câncer de mama, é o único já reconhecidamente eficaz na redução da mortalidade pela neoplasia mamária, em especial na pós-menopausa ${ }^{1,2}$.

Este exame é utilizado de forma sistemática nos programas de rastreamento do câncer de mama para mulheres acima de 40 ou de 50 anos, de acordo com cada programa de rastreamento ${ }^{2}$. Ainda, para as mulheres no climatério, mesmo com a redução de sua eficácia nas usuárias de terapia hormonal (TH), a mamografia tem sido um exame adequado para avaliar as mamas nesse grupo de mulheres ${ }^{3}$. Já para as mulheres com sintomas mamários, ela também tem sido utilizada após os 35 anos ou, então, independentemente da idade para aquelas que se apresentam com lesões clinicamente suspeitas de malignidade ${ }^{4}$.

Não obstante, um grande número de mulheres apresenta desconforto, que varia de discreto a insuportáve $e^{5-8}$, sendo que após o exame, os traumas físico e psicológico deixados podem levar as mulheres a evitar exames futuros ou, ainda, a comentar com amigas, impedindo que estas, por sua vez, se submetam a este importante método propedêutico ${ }^{5,9}$.

Assim, o presente estudo teve como objetivo conhecer a freqüência de desconforto e de dor ocasionada pela realização da mamografia e identificar fatores relacionados a estes sintomas.

\section{Métodos}

Após aprovação pelo Comitê de Ética em Pesquisa do Hospital das Clínicas da Universidade Federal de Goiás, foram incluídas nesse estudo 2.164 mulheres, sendo 996 previdenciárias, tendo realizado a mamografia no Hospital Araújo Jorge, e I 68 que realizaram o exame na rede privada. Todas as participantes tinham solicitação de mamografia, decorrente de rastreamento ou de investigação de alterações mamárias.

Após as explicações cabíveis e tendo assinado o termo de consentimento informado, a mulher recebia dois formulários. $O$ primeiro abordava questões relacionadas à própria paciente e era preenchido antes do exame. O segundo incluía a escala analógica visual de dor e abordava o desconforto ocasionado pelo exame, o qual era preenchido após a mamografia. Não havia em nenhum dos formulários alguma forma de identificação pessoal da mulher.

Em cada formulário havia um número de entrada no estudo, que foi usado para a identificação e casamento dos dois formulários. Os formulários foram entregues pela recepcionista de cada clínica, individualmente, a cada paciente e eram auto-explicativos. Depois de preenchidos, os mesmos foram arquivados com um funcionário pré-determinado por cada centro participante, periodicamente recolhido por um dos membros do estudo e digitados em banco de dados. 
FREITAS JÚNIOR R ET AL.

\begin{tabular}{|c|c|c|c|c|}
\hline Variável & Categorização & Média de dor & Teste $\mathrm{t}$ & $\mathrm{p}$ \\
\hline \multirow[t]{2}{*}{ Local do exame (tipo de paciente) } & Previdenciária & 3,4 & 9,34 & 0,000001 \\
\hline & Privada & 4,3 & & \\
\hline \multirow[t]{2}{*}{ Idade } & Até 50 anos & 4,0 & 2,87 & 0,005 \\
\hline & $>50$ anos & 3,7 & & \\
\hline \multirow[t]{2}{*}{ Mastalgia prévia } & Não & 3,7 & 8,34 & 0,000001 \\
\hline & $\operatorname{Sim}$ & 4,8 & & \\
\hline \multirow[t]{2}{*}{ Uso de ACO } & Não & 3,8 & 2,67 & 0,01 \\
\hline & Sim & 4,2 & & \\
\hline \multirow[t]{2}{*}{ Uso de TH } & Não & 3,8 & 1,32 & 0,2 \\
\hline & Sim & 4,0 & & \\
\hline
\end{tabular}

Os exames mamográficos foram feitos em quatro centros: Setor de Radiologia do Hospital Araújo Jorge (pacientes previdenciárias), Clínica São Camilo, Clínica Radiológica de Goiânia e Clínica ARO, sendo os três últimos serviços privados.

\section{A mamografia}

Os exames mamográficos foram realizados em ambas as mamas de cada voluntária, sempre em duas incidências (médio lateral oblíqua e crânio-caudal). No Serviço de Radiologia da ACCG, foi utilizado um aparelho da marca Villa Sistemi Medicalli, modelo Vênus HS, já nas demais clínicas utilizou-se aparelhos GE, modelo 600T.

Em todos os exames, o técnico de radiologia comprimia a mama da paciente de acordo com a necessidade, visando obter a melhor imagem possível, porém, sempre respeitando individualmente o quanto a paciente suportava de compressão. Assim, não foi estabelecida para o estudo uma compressão uniforme, mas aquela necessária para uma leitura adequada do filme, de acordo com e experiência de cada clínica.

\section{Variáveis dependentes}

Dor: A avaliação da dor foi feita por meio de escala linear analógica, variando de 0 a 10 para as mulheres que não sentiram dor e para as que sentiram dor intensa, respectivamente.

Desconforto: Essa avaliação foi feita pela paciente e qualificada em quatro categorias: sem desconforto, desconfortável mas tolerável, muito desconfortável ou intolerável.

\section{Variáveis independentes}

Idade da paciente na realização do exame mamográfico; centro de acordo com o local em que a paciente fez a mamografia e depois subdividido em clínicas privadas ou serviço público. Realização de mamografia prévia se sim ou se não. Presença de mastalgia prévia, qualificada se a paciente não apresentava mastalgia; se ela apresentava mastalgia, porém, ocasionalmente; se a mastalgia era freqüente, mas suportável; ou se a mastalgia, além de freqüente, era intensa ou insuportável.

Uso de contraceptivos orais (ACO): para as mulheres que estavam no menacme, foram consideradas usuárias de pílula aquelas que estavam usando a medicação por ocasião do exame mamográfico, e como não usuárias as que não faziam uso da medicação na data do exame.

Uso de terapia hormonal $(\mathrm{TH})$, sendo consideradas como usuárias as mulheres que faziam uso da medicação por ocasião do exame e como não usuárias aquelas que não faziam o uso. Ao final foi questionado se a mulher faria novas mamografias no futuro com a possibilidade de resposta positiva ou negativa.

\section{Análise estatística}

Os dados foram digitados no Banco de Dados Dbase III + e, após dupla checagem, foram analisados, usando o teste do Qui-quadrado ou o teste $t$ de Student, quando aplicáveis. Para a análise univariada, foram calculados o odds ratio e o intervalo de confiança de cada variável, sempre considerando o desconforto da paciente. Também foi conduzida uma análise multivariada para o desconforto, verificando a interdependência entre elas. Considerou-se como significante quando o valor de $p$ foi igual ou inferior a 0,05 .

\section{Resultados}

Das 2164 pacientes incluídas no estudo, 996 (46\%) foram submetidas a mamografia no Hospital Araújo Jorge (pacientes previdenciárias) e as demais 1.168 nas clínicas privadas participantes. A média de idade das pacientes foi de 47,9 anos, com desvio padrão de 10,4 anos. Ainda com relação à idade, 534 pacientes tinham menos de 40 anos, 820 de 41 a 50 anos e 798 acima de 50 anos (a idade não foi preenchida na ficha de 12 pacientes). Para I.I80 pacientes, aquela era a primeira mamografia e as demais já haviam sido submetidas a pelo menos um exame anteriormente.

A análise da média de dor referida pela paciente, usando a escala analógica visual para cada um dos possíveis fatores associados, é apresentada na Tabela I.

A Figura I mostra a taxa percentual de desconforto referido pela paciente durante a mamografia. De acordo com a análise multivariada, os fatores que estiveram associados ao desconforto durante a realização do exame foram o local de realização do exame, no qual o desconforto intenso ou intolerável foi referido 


\begin{tabular}{|c|c|c|c|}
\hline Fator & Categorização & OR (95\% IC) & $p$ \\
\hline Idade(anos) & $\begin{array}{l}>50 \\
\text { Até } 50\end{array}$ & $\frac{1}{1,29(0,71-2,03)}$ & 0,40 \\
\hline Local de realização (tipo de paciente) & $\begin{array}{l}\text { Previdenciária } \\
\text { Privada }\end{array}$ & $\frac{1}{2,50(1,64-5,17)}$ & 0,01 \\
\hline Mamografia prévia & $\begin{array}{l}\text { Não } \\
\text { Sim }\end{array}$ & $\begin{array}{c}1 \\
1,19(0,82-2,27)\end{array}$ & 0,359 \\
\hline Mastalgia prévia & $\begin{array}{l}\text { Não } \\
\text { Sim }\end{array}$ & $\frac{\mid}{3,15(1,96-7,12)}$ & 0,01 \\
\hline Uso de ACO & $\begin{array}{l}\text { Não } \\
\text { Sim }\end{array}$ & $\begin{array}{c}1 \\
1,12(0,73-2,08)\end{array}$ & 0,596 \\
\hline $\mathrm{TH}$ & $\begin{array}{l}\text { Não } \\
\text { Sim }\end{array}$ & $\frac{\mid}{\mid}$ & 0,284 \\
\hline
\end{tabular}

$\mathrm{OR}=$ odds ratio; $I \mathrm{C}=$ intervalo de confiança; Previdenciária = paciente que realizou a mamografia no Hospital Araújo Jorge; Privada = paciente que realizou a mamografia em uma das clínicas privadas; $\mathrm{ACO}=$ anticoncepcional oral; $\mathrm{TH}=$ terapia hormonal

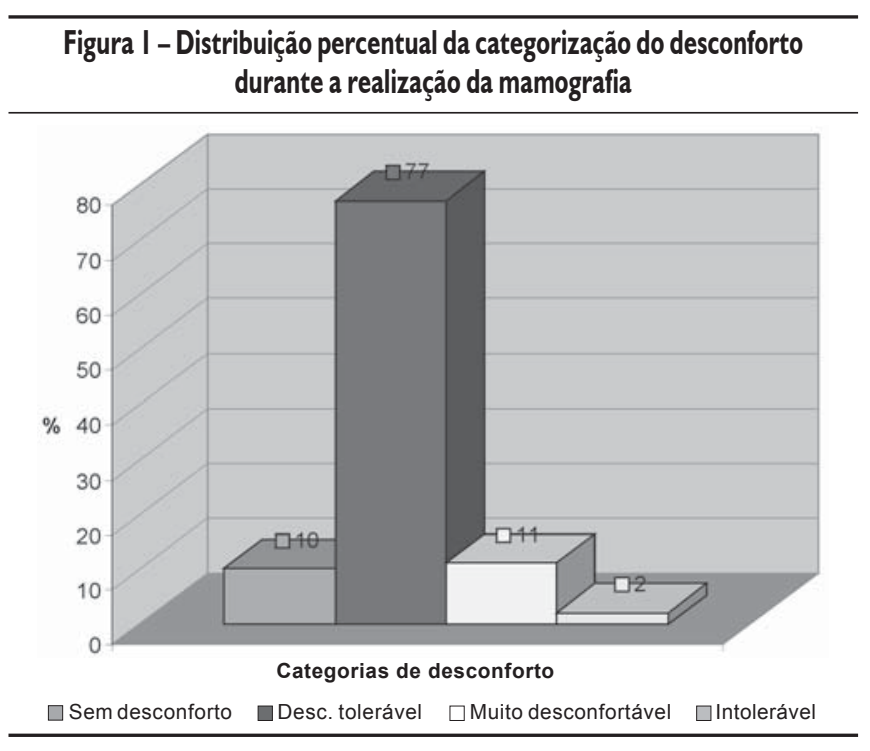

duas vezes mais pelas pacientes do setor privado do que pelas que fizeram a mamografia no Hospital Araújo Jorge. As pacientes que apresentavam história de mastalgia prévia referiram desconforto intenso ou intolerável três vezes mais que as que não apresentavam esse sintoma previamente (Tabela 2). Apenas 42 pacientes $(2 \%)$ referiram que recusariam fazer nova mamografia no futuro e esse fato foi altamente associado àquelas para quais o exame foi muito desconfortável ou intolerável.

\section{Discussão}

A causa primária da dor e do desconforto físico durante a mamografia é a compressão exercida sobre a mama pelo prato plástico de compressão e o porta filme ${ }^{10}$. O exame depende muito de uma compressão efetiva, que interfere diretamente na resolução mamográfica, reduzindo a imprecisão e melhorando a qualidade do contraste das imagens ${ }^{11,12}$.
Apesar da grande maioria das pacientes ter referido dor e desconforto durante a mamografia, deve ser salientado que apenas $2 \%$ referiram desconforto intolerável. Esse fato mostra claramente que, apesar de ser um exame desagradável, a mamografia foi bem tolerada pela quase totalidade do grupo estudado. Nossos números ratificam trabalhos anteriores que mostram que o desconforto intolerável acontece em $0,2 \%$ a $3 \%$ de todas as mulheres submetidas a mamografia $6,7,13$.

Observamos que as mulheres que realizaram a mamografia nas clínicas privadas apresentaram uma quantificação maior de dor do que aquelas que realizaram o exame no serviço previdenciário. Esse fato pode ser explicado pela possível diferença do limiar de dor, a partir do princípio que as mulheres previdenciárias, com menor poder aquisitivo, por apresentarem um sofrimento maior em suas vidas, têm um limiar de dor maior e uma menor expectativa em relação à sensação de dor, em si.

Quanto à idade, não foi surpresa que as pacientes mais jovens referissem uma média de dor maior do que as mais idosas ${ }^{14}$. Isso, possivelmente, está relacionado com outro fato, também observado por nós, de que as mulheres que apresentavam mastalgia prévia, causada por alteração funcional benigna da mama ${ }^{15}$, também referiram uma quantificação maior de dor do que aquelas que não apresentavam mastalgia. Essa relação é compartilhada por outros estudos

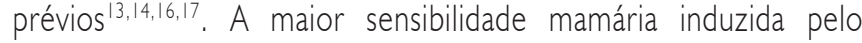
contraceptivo hormonal também poderia explicar uma quantificação maior de dor para as usuárias correntes dessa medicação.

Em relação à terapia hormonal, apesar de estudos mostrarem que as mamas ficam mais sensíveis em usuárias ${ }^{18}$, nosso estudo não mostrou diferença significante da dor e do desconforto para as mulheres que usam ou não a reposiçãa hormonal.

Quanto ao desconforto, após análise multivariada, nos serviços privados as mulheres apresentaram 2,5 vezes mais chances de terem dor que as mulheres previdenciárias, as quais fizeram a mamografia no Serviço do Hospital Araújo Jorge. Ainda por essa análise, a mastalgia prévia também foi confirmada como fator independente, associada com o maior desconforto durante a realização da mamografia, sendo 
Freitas JÚNIOR R ET AL.

que as pacientes que apresentaram mastalgia prévia tiveram três vezes mais chance de ter um desconforto acentuado à mamografia que aquelas que não apresentam mastalgia.

\section{Conclusão}

Esse trabalho mostra que a dor e o desconforto durante a mamografia acontecem com grande freqüência, principalmente nas pacientes que apresentam mastalgia. Assim, devem ser tentadas ações no sentido de diminuir a dor e o desconforto ao exame mamográfico, de forma que a mulher possa usufruir dessa tecnologia em seu benefício, porém, sem sofrimento.

\section{Conflito de interesse: não há}

\section{SUMMARY}

\section{DISCOMFORT AND PAIN DURING MAMMOGRAPHY}

INTRODUCTION. Mammography is the mostimportantmethodfor early detection of breast cancer, however, patients frequently complain of discomfort and pain.

OBIECTIVE. Evaluate how often discomfort and pain are felt during mammography and identify factors that may be associated to these complaints.

METHODS. A prospective study including 2, 164 patients recruited from public (996) and private $(1,168)$ health services was carried out. After the imaging procedure, patients quantified pain using a linear analogical scale. Discomfort was qualitatively evaluated by multivariate analysis.

RESULTS. Discomfort was reported by $90 \%$ of the patients, and of these $12 \%$ rated the sensation as intense or intolerable. Only $2 \%$ of the women in the study group did not feel any pain during the procedure. Factors associated to pain were: age, use of birth control methods (BCM), previous mastalgia and patients from the private health sector. Discomfort was independently associated to the service where the procedure was performed $(O R=2.50 \mathrm{IC} 1.64-5.17)$ and to previous mastalgia $(O R=3.15 \mathrm{IC} 1.96-7.12)$. When asked about a repeat mammography, $98 \%$ of the patients said they would comply.

CONCLUSION. Young women ( $<50$ years), BCM users, mastalgia patients and women who were using the services of a private clinic felt more intense pain and discomfort during mammography. [Rev Assoc Med Bras 2006; 52(5): 333-6]

KEY wORDS: Breast. Mammography. Cancer. Screening. Pain. Discomfort.

\section{REFERÊNCIAS}

I. Tabar L, Fagerberg G, Chen HH, et al. Efficacy of breast cancer screening by age. Cancer 1995;75:2507-17.

2. Kerlikowske K, Grady D, Rubin SM, Sandrock C, Ernster VL. Efficacy of screening mammography. A meta-analysis. JAMA 1995;273: | 49-54.

3. Warren R. Hormones and mammographic breast density. Maturitas 2004:49:67-78.

4. França JB, Freitas Jr R, De Conti RC, Philocreon GR. Conduta diagnóstica nos nódulos palpáveis da mama: Rotina do Departamento de Ginecologia da Universidade Federal de Goiás. Femina 1993;21:932-43.

5. Baines CJ, To T, Wall C. Women's attitudes to screening after participation in the National Breast Screening Study. Cancer 1990;65:1663-9.

6. Bennett IC, Osborne JM, Baker CA. Discomfort during mammography: A survey of women attending a breast screening center. Breast Dis 1994;7:35-41.

7. Petti DA, Hotta EH, Pinheiro JRV, D'Angelo LF, Oliveira LD, Melo PT, et al. Mamografia e dor: um mito ou um fato? An Paul Med Cir 1998; 125: 48.

8. Kashikar-Zuck S, Keefe FJ, Kornguth P, Beaupre P, Holzberg A, Delong D. Pain coping and the pain experience during mammography: a preliminary study. Pain 1997;73: 165-72.

9. Elwood M, McNoe B, Smith T, Bandaranayake M, Adan H, Doyle TCA. Once is enough: why some women do not continue to participate in a breast cancer screening programme. N Z Med J 1998; | I 1: 180-3.

10. Prue LK. Atlas of mammographic positioning. Philadelphia: Saunders Co; 1994.

II. Jackson VP, Lex AM, Smith DJ. Patient discomfort during screen-film mammography. Radiology 1988; 168:42I-3.

12. Poulos A, McLean D, Rickard M, Heard R. Breast compression in mammography: how much is enough? Australas Radiol 2003;47: 121 -6.

13. Sullivan DC, Beam CA, Goodman SM, Watt DL. Measurement of force applied during mammography. Radiology 1991; 181:355-7.

14. Gupta R, Nayak M, Khoursheed M, Roy S, Behbehani Al. Pain during mammography: impact of breast pathologies and demographic factors. Med Princ Pract 2003; I 2: 180-3.

15. Barros ACSD, Nazário ACP. Alterações funcionais benignas das mamas. Barros ACSD, Silva HMS, Dias EN, Nazário ACP, Figueira Filho ASS. Mastologia: condutas. Rio de Janeiro: Revinter; 1999. p.28-33.

16. Stomper PC, Kopans DB, Sadowsky NL, Sonnenfeld MR, Swann CA, Gelman RS, et al. Is mammography painful? A multicenter patient survey. Arch Intern Med 1988; | 48:52 I-4.

17. Sapir R, Patlas M, Strano SD, Hadas-Halpern I; Cherny NI. Does mammography hurt? J Pain Symptom Manage 2003;25:53-63.

18. Hughes LE, Mansel RE, Webster DJT. Breast pain and nodularity. Hughes LE, Mansel RE, Webster DJT. Benign disorders and diseases of the breast. $2^{\text {nd }}$ ed. London: Saunders Co; 2000. p.95-121.

Artigo recebido: 25/07/2005

Aceito para publicação: 18/10/2005 